\title{
The PANDAS Controversy: Why (and How) Is It Still Unsettled?
}

\author{
Tanya K. Murphy • Diana M. Gerardi • \\ E. Carla Parker-Athill
}

Published online: 16 August 2014

(C) Springer International Publishing Switzerland 2014

\begin{abstract}
Acute-onset OCD and/or tics in children, known by the term Pediatric Autoimmune Neuropsychiatric Disorders Associated with Streptococcus (PANDAS), has been a focus of pediatric developmental and psychiatric research for nearly three decades. Studies have suggested that this condition results from molecular mimicry, in which anti-basal ganglia antibodies target brain proteins. While the pathogenesis of this condition is still widely debated, the clinical phenotype has shown consistent features and evidence that the dramatic symptoms cause substantial impairment in the child and in family functioning.
\end{abstract}

Keywords PANDAS $\cdot$ PANS $\cdot$ OCD $\cdot$ Tics $\cdot$ Streptococcal infections

\section{Introduction}

Pediatric autoimmune neuropsychiatric disorder associated with streptococcus (PANDAS) is a proposed subtype of obsessive-compulsive disorder (OCD) and/or tic disorders characterized by an abrupt, dramatic pattern of episodic or saw-tooth symptoms, with the initial episode occurring acutely or "overnight." Over a period of 24 to 48 hours, parents of children with PANDAS report their child as experiencing OCD and/or tics, as well as cognitive decline and behavioral regression, including attention difficulties, deterioration in

T. K. Murphy $(\bowtie) \cdot$ D. M. Gerardi • E. C. Parker-Athill

Division of Pediatric Neuropsychiatry, Rothman Center, Department

of Pediatrics, University of South Florida, 880 6th St. South, Suite

460, Box 7523, St. Petersburg, FL 33701, USA

e-mail: tmurphy@health.usf.edu

T. K. Murphy

Department of Psychiatry, University of South Florida, Tampa, FL, USA handwriting and other fine-motor skills, nighttime fears, and occasionally psychosis. Frequent urination and choreiform movements have also been reported. A parent describing their child as "a changed child" is not uncommon. While the exact cause of this phenotype has not been established, the most frequently reported association has been a group-A streptococcal (GAS) infection. This review provides an update of the recent literature published on PANDAS.

\section{PANDAS Iterations}

Since Swedo and colleagues proposed the initial PANDAS criteria [1], the validity of the PANDAS diagnosis has been a subject of controversy in the medical professional community $[2,3]$. After a group of researchers familiar with PANDAS presented data on youth with these neuropsychiatric symptoms, the term pediatric acute-onset neuropsychiatric syndrome (PANS) was established by one group [4・•]; while another proposed childhood acute neuropsychiatric symptoms

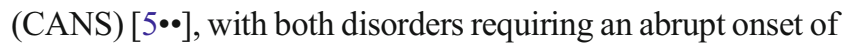
neuropsychiatric symptoms in a pediatric patient. As temporal relationships to GAS infections are often difficult to establish, these proposals allow for the possibility that other pathogens, as well as other medical conditions, may be involved in the pathophysiology of this abrupt neuropsychiatric presentation. Therefore, neither the PANS nor CANS diagnosis is contingent upon a criterion of temporal association with a GAS infection. In addition, the PANS criteria differs from that of PANDAS in that the former notes that the child could present with acute-onset severely restricted food intake. Both PANS and CANS should be viewed as appropriate steps toward the iterative process to better define this acute neuropsychiatric presentation. However, neither approach proposes how to address presentations that are on the 'spectrum' of symptoms and severity. For example, the CANS proposal outlines a 
comprehensive diagnostic assessment, but it is not clear which tests are needed for a specific symptom or severity level. In contrast with PANDAS, both approaches have de-emphasized tics as a core presenting symptom.

\section{PANDAS Characteristics}

Research has continued to aim to solidify the clinical characteristics of a patient with PANDAS. In addition to OCD and tics, hyperactivity, inattention, psychosis, separation anxiety, behavioral regression, nighttime fears and rituals, decline in school performance, sensory issues, and oppositional behaviors all continue to be reported as prevalent in PANDAS [1, 6,

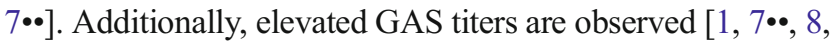
9], as well as improvement in symptoms while on antibiotics $[1,7 \bullet \bullet, 10]$. Family history also plays a role, as high rates of maternal autoimmunity [11] or family members with OCD/ tics or other neurological disturbances have been reported [12, 13]. Environmental factors also appear to be important, as significant variations in presentations have been found among youth with PANDAS and their identical siblings [14]. Similarly, in two siblings with PANDAS, one child exhibited tics and the other child had tics and comorbid OCD, and both presentations were associated with a GAS infection [15]. Regarding the timing of other common symptoms, such as hyperactivity, urinary urgency, impulsivity, handwriting deterioration, separation anxiety, and declining school performance, these often occur during the initial PANDAS episode [6]. Later episodes have been characterized by worsening inattention, mood lability, bedtime fears and rituals, and oppositional behaviors [6].

Comparisons between PANDAS OCD and non-PANDAS OCD have been investigated, and clinical differentiations are being established. In particular, attention deficit hyperactivity disorder (ADHD) symptoms and psychosis have been found to be more common in youth with PANDAS OCD than in peers with non-PANDAS OCD $[6,7 \bullet \bullet$. Findings regarding the prevalence of separation anxiety in PANDAS versus nonPANDAS OCD are mixed $[6,7 \bullet \bullet]$. Youth with PANDAS have been more likely to exhibit a dramatic onset of symptoms with definite remissions, a history of tonsillectomies and/or adenoidectomies, evidence of GAS infection, and clumsiness $[7 \bullet \bullet]$. Total tics and vocal tics have been found to be more severe in children with PANDAS [6, 7••] (see Table 1 for symptoms reported in the PANDAS phenotype). Neurocognitive functioning in PANDAS has also been investigated and significant impairment in visuospatial recall has been observed [16]. Although all youth demonstrated average to above-average performances in tests of academic achievement and recognition memory, elevations in anti-streptolysin $\mathrm{O}$ (ASO) titers correlated with poor performance on immediate and delayed visual-spatial memory, as well as fine motor speed tests. Youth with PANDAS have also been found to have poor response accuracy in a test of attention and suppression compared to healthy controls [17], a finding consistent with the prevalence of ADHD symptoms found in youth with PANDAS [1, 6, 7••]. However, the course of PANDAS OCD is the primary distinguishing feature from "classical" OCD, as both phenotypes share significant overlap.

\section{PANDAS Etiology}

\section{Infectious Triggers}

While much debate continues surrounding PANDAS, strong evidence exists for the role of infection in precipitating the symptoms of tics and OCD observed in these patients. In 1965, Langlois and Force described the clinical presentation of a 6-year-old patient who developed tic symptoms after recurrent infections, which were subsequently treated with antibiotics [18]. More recent studies by other groups, including Swedo in the initial characterization of the PANDAS phenotype, have presented evidence for an association of GAS infection and symptom exacerbation. In Swedo's case series, GAS infection and/or exposure were found to be the inciting trigger for symptom onset and/or exacerbation [1]. Another study prospectively examining children with tics or OCD found that those with an episodic presentation of OCD and tic symptoms were more likely to have chronically elevated streptococcal titers compared to patients with remitting or stable symptoms [9]. Positive findings regarding a correlation between titer levels and OCD severity [9], as well as with tic severity [19, 20], have been found. Although not always found [21-23], patients with Tourette syndrome (TS) have been observed to have higher levels of ASO and/or antiDNase B titers compared to controls [24•, 25]. Despite the positive findings of these studies, others have reported no correlation between GAS infection and $\mathrm{OCD} / \mathrm{tic}$ symptomology [26-29]. Notably, while no correlation between OCD and/or tic exacerbation and GAS infection was found [28], youth with tics or OCD had been found to have more GAS infections per year than healthy controls [24•, 28]. In addition, an increase in the incidence of tics has been reported as occurring in temporal proximity to an outbreak of streptococcus in a pediatric clinic [30]. PANDAS symptom severity and recurrence of episodes has also been correlated with the number of past GAS infections [10]. Notably, patients with OCD or tics have been more likely than healthy controls to have had a GAS infection within the three months prior to onset of symptoms [8].

GAS infection occurrence and correlation with OCD and/ or tic exacerbations continues to be investigated [24•, 26-29]. In most of these longitudinal studies, neither a greater occurrence of GAS infections, nor GAS-related clinical 
Table 1 PANS Phenotype

\begin{tabular}{llll}
\hline PANDAS Characteristics & $\begin{array}{l}\text { Swedo et al. 1998 [1] } \\
\mathrm{N}=50\end{array}$ & $\begin{array}{l}\text { Bernstein et al. 2010 [6] } \\
\mathrm{N}=21\end{array}$ & $\begin{array}{l}\text { Murphy et al. 2012 [7••] } \\
\mathrm{N}=41\end{array}$ \\
\hline Male prevalence & $72 \%$ & $67 \%$ & $68 \%$ \\
OCD & $86 \%$ & $100 \%$ & $88 \%$ \\
Tics & $80 \%$ & $33 \%$ & $81 \%$ \\
ADHD & $40 \%$ & $78 \%$ & $61 \%$ \\
Affectivity instability & $66 \%$ & $84 \%$ & $59 \%$ \\
Psychosis & $0 \%$ & $0 \%$ & $12 \%$ \\
Separation anxiety & $46 \%$ & $74 \%$ & $29 \%$ \\
Enuresis & $12 \%$ & $42 \%$ & $24 \%$ \\
Choreiform & $50 \%$ & $42 \%$ & $78 \%$ \\
Frequent urination & - & $26 \%$ & $42 \%$ \\
Handwriting deterioration & $36 \%$ & $61 \%$ & $29 \%$ \\
\hline
\end{tabular}

exacerbations were found in children with OCD and/or tics. However, despite these studies being performed at multiple centers, the potential variation of PANDAS cases and interrater reliability were never addressed; in one study many in the PANDAS group were treated with antibiotics by their primary care providers [27]. Many of these prospective studies appear to feature more of a tic-dominant phenotype rather than an acute-onset OCD phenotype. Additionally, many studies may not correctly examine the temporal association to GAS infection, or the duration between infection and/or exposure, and symptoms onset. Therefore, the generalizability of these results is questionable, and appears to presume that all future flares will be GAS-related. However, not all symptom flares, especially tic-related, are likely to have GAS as the only trigger. This has been demonstrated in Sydenham's chorea (SC) [31], a prototypical infection-triggered disease that served as the original diagnostic model for PANDAS [32]. Also, until reliable biological markers to differentiate PANDAS from non-PANDAS presentations have been established, it is also possible that some children in the "nonPANDAS' groups will flare with GAS or other infections.

Different presentations of GAS infections, exposures by family members, and infections at sites other than the pharynx need further study. A GAS pharyngitis diagnosis can be missed in preschool children, as their symptoms often include gastroenteritis as well as less tonsillar exudates and cervical adenopathy than is observed in school-aged children [33]. Asymptomatic carriers of GAS must also be considered, as up to $20 \%$ of school-aged children are asymptomatic GAS carriers, and $25 \%$ of family members of a child with GAS are actually asymptomatic carriers [34]. Regarding streptococcus at other sites, a recent case report documented perianal streptococcal dermatitis as the precipitating factor to a PANS initial episode and/or exacerbation in three children [35]. Interestingly, two of these children had a history of pharyngeal streptococcal infections, and research has suggested that pharyngeal strains of GAS can migrate to other areas of the body [36, 37].

Infectious triggers other than GAS have also been considered. For example, preceding upper respiratory infections (URI) have also been correlated with tic severity [38], and viral infections have been reported as preceding acute-onset OCD and tics in children [39]. In adults, cases of new-onset tics after a URI have been reported [40]. Mycoplasma pneumonia, the bacteria associated with "walking pneumonia," has been implicated in the development of neurologic sequelae [41]. Patients with TS have been found to have higher mycoplasma pneumonia titers and are more likely to be immunoglobulin (Ig) A positive compared to controls [42]. Cases of tic exacerbations following a mycoplasma pneumonia infection have also been reported [20]. Due to symptomatic overlap between PANDAS and Lyme disease, a recent literature review attempted to juxtapose the two conditions [43]. Notably, Lyme disease has been reported in the medical history of those with OCD [44], and also in a child with Tourette syndrome, who experienced partial symptom alleviation after eradication of Borrelia burgdoferi [45]. However, the issue of neuropsychiatric symptoms secondary to Lyme disease is not without controversy, and further research is needed on the role of Lyme disease in youth with PANDAS.

Autoimmune Implications

Autoimmune reactions have been proposed to be at the center of PANDAS pathology. Immune abnormalities have been frequently observed in PANDAS patients [46-48], including findings analogous to those seen in SC, such as evidence of GAS-mediated anti-neuronal autoantibodies, dopamine receptor autoantibodies, and basal ganglia pathology [49, 50]. Three putative antibodies that have been implicated in SC, including $\mathrm{N}$-acetyl-beta-D-glucosamine (a streptococcal group-A carbohydrate antigen), tubulin, and dopamine 2 
receptor (D2R), are of interest in PANDAS research. Several groups have reported evidence of these autoantibodies within the PANDAS population, with additional evidence of reactivity with basal ganglia neurons [46-48]. Additionally, these anti-neuronal proteins were found to be specific to the PANDAS population, as patients with a previous GAS infection did not demonstrate a significant serological presence of these autoantibodies [47].

Despite these findings, and strong clinical similarities with $\mathrm{SC}$, there is still much debate as to the validity of this mechanism in PANDAS, as several groups have presented evidence disputing an autoantibody mediated mechanism, with findings of no differences in the level of anti-neuronal or D2R antibodies in the sera of PANDAS patients when compared to those with Tourette syndrome or control individuals [51-53]. However, these differing observations do not preclude the possibility of an autoimmune-mediated disease mechanism, as methodological differences, particularly in patient characterization, often account for a significant level of variability in study outcomes. While some studies have used PANDAS cohorts that meet the diagnostic criteria proposed by Swedo, others have had mixed populations that do not meet PANDAS criteria, being largely comprised of tic patients without features of comorbid OCD [51-53]. Several studies, conducted in patients with various presentations of tic disorders (both with and without comorbid OCD), have noted significant differences in the immunological profiles of these groups; one study noted differences in cytokine expression in patients who presented with tics only, compared to those who presented with tics and OCD [54]. Additionally, medication status, including complementary alternative medicine, and duration of illness, significantly affect study outcomes, particularly the comparison of immune profiles and immunological characteristics. These factors need to be carefully considered when comparing acute illnesses, like SC and acute disseminated encephalomyelitis, with chronic illnesses like TS and OCD and even more chronic presentations of PANDAS.

Nevertheless, other studies have provided strong evidence of abnormal immunological responses in the PANDAS population, which may suggest a predisposition to autoimmune reactions to GAS. In a study of tic patients compared to patients with a previous history of GAS pharyngitis, tic patients were shown to have a stronger immune response to GAS antigens, as well as preferential and specific reactions to GAS antigens, not seen in controls or patients with GAS pharyngitis [55]. Further evidence for autoimmune reactions within the PANDAS population may be found in the association of TNF- $\alpha$ gene polymorphisms with OCD, a key feature of PANDAS pathology, which has been shown to increase susceptibility and define clinical outcomes [56, 57]. A recent study found TNF- $\alpha-308$ G/A polymorphism in youth with PANDAS but not in controls [58]. TNF- $\alpha$ abnormalities in patients with $\mathrm{OCD} / \mathrm{tics}$ and an association with symptom course further support a potential predisposition toward an autoimmune phenotype, as TNF- $\alpha$ has been repeatedly correlated with autoimmune disorders [59]. In addition to its proinflammatory properties, TNF- $\alpha$ also functions as an immune regulator and has been shown to control monocyte activation and T cell activity [60]. These interactions may be important in explaining the infection-mediated symptom course, as the inability of monocytes to differentiate into macrophages and effectively clear bacterial pathogens may lead to chronic inflammatory states and autoimmune reactions [61]. A recent study that investigated immune abnormalities in PANS patients also demonstrated lower spontaneous production of proinflammatory and counter-regulatory cytokines compared to controls. In addition, peripheral blood mononuclear cells of PANS patients produced less pro-inflammatory cytokines than those of controls [62]. These results support innate immune dysregulation as a diathesis to infections and/or autoimmune diseases in individuals with PANDAS [63].

\section{Animal Models}

Current PANDAS models have focused on the immune hypothesis of PANDAS by utilizing GAS immunization or passive transfer of GAS antigens to naïve animals to recreate the core symptoms seen in PANDAS [64・ 65,66$]$. Both approaches have yielded models displaying behavioral, pharmacological, neuropathological and immunological phenotypes seen clinically in PANDAS. Neurophysiological observations have included basal ganglia pathologies, including antibody deposition and autoantibodies to neuronal and dopamine receptor proteins; behavioral observations have replicated the motor dysfunction, evident by deficits in motor coordination, including impaired food manipulation and beam walking, and compulsions and anxiety-like behaviors, such as increased induced-grooming, which are central to PANDAS pathology $[64 \cdot, 67]$. Perhaps the most significant contribution of these models has been the ability to clearly delineate a relationship between GAS infection and resultant pathological outcomes as it is an important step toward understanding the role of GAS, and resultant autoimmune reactions to GAS infection, in the neuropathology and behavioral phenotypes observed clinically in PANDAS. A second group of models have utilized a different approach by targeting the role of CNS proteins in OCD and/or tic symptomology. These models help to identify gene targets through the use of genetically modified animals. Models involving deletions of CNS protein, such as the dopamine receptors (a common target for GAS-induced autoantibodies), examine the role of these proteins in PANDAS pathology and may help characterize the underlying mechanism for the autoimmune reactions seen in PANDAS [68]. In addition to shedding light on the 
pathological mechanism of this disorder, PANDAS models have also revealed potential targets for therapeutic intervention, with evidence of attenuation of PANDAS symptomology, particularly neuropsychiatric and behavioral symptoms, by IgG depletion and D2R blockers [64•]. In a mouse model for Sydenham's chorea, it was found that IgG from SC and PANDAS with small choreiform movements exhibited a reaction with D2R in ELISA [67]. Distinguishing SC from PANDAS was evident in FLAG-tagged D2R, and sera from both SC and PANDAS induced inhibition of D2R signaling on transfected cells that were comparable to dopamine. Overall, these results suggest a mechanism by which antibodies alter the brain and result in movement and neuropsychiatric disorders. Another study using a rat model of SC found similar results, in that rats exposed to GAS antigens began displaying motor symptoms and compulsive behaviors, with symptom alleviation occurring after haloperidol and paroxetine treatment [64•]. The GAS exposure resulted in antibody manifestations, specifically autoantibodies against D1R and D2R, and neurochemical alterations consistent with those that occur in SC. Calcium/calmodulin dependent protein kinase II elevations were also found [64•], and have been found in the sera of patients with SC and other neuropsychiatric disorders [69]. These models are an important tool in understanding the etiology and pathological mechanism of PANDAS, as well as uncovering targets of therapeutic intervention.

\section{Varieties in Presentation}

Cases of PANDAS with comorbid Kleine-Levin syndrome [70], and hyperimmunogloblin D syndrome [71] have been recently reported. Conversion disorder [72] has also been noted. Clinically, it is not uncommon to see an akathisia presentation in children with suspected PANDAS, manifested by the child complaining of needing to move their arms and legs, with the movements subsequently labeled as a conversion disorder. Interestingly, cases of GAS-triggered anorexia with symptom remittance following antibiotic therapy have been reported [73-75]. Food restrictive behaviors have been noted in youth with PANDAS [6], and anorexia is a common comorbidity in OCD [76]. In addition, acute-onset stuttering has been reported after a documented GAS infection $[14,77]$.

\section{Case Vignette}

The patient is a 4-year-old girl who presented with acute-onset OCD and vocal tics that developed in temporal proximity to a URI. Her history is significant for two prior episodes, each lasting approximately two weeks, in which she exhibited mild motor tics that did not cause significant impairment. These episodes occurred 12 months and six months prior to the evaluation. Her recent episode, which was described as causing significant impairment, occurred two months prior to evaluation. One month prior to the episode, the patient developed a fever and was diagnosed with a URI, for which she received azithromycin $250 \mathrm{mg}$ daily for five days. One month later, she began to exhibit new-onset symptoms of OCD, including reassurance seeking and compulsive hand washing. New-onset deterioration in handwriting, mild oppositionality, and vocal tics also emerged. Symptoms of ADHD, sleep disturbance, separation anxiety, changes in appetite, and sensory issues were denied. Developmental milestones were within normal limits, and medical history was significant for frequent URIs and otitis media. At the evaluation, the patient yielded a positive rapid GAS test and was diagnosed with OCD and a rule-out of chronic tic disorder. She was prescribed a 10day course of azithromycin. At one-month follow-up, the parents reported the patient as exhibiting a rapid response to the antibiotic, with her symptoms having decreased $70 \%$. She exhibited less reassurance seeking and almost no anxiety. Notably, her brother had perianal GAS and the patient had been exposed to GAS pharyngitis by a classmate. She did not exhibit elevated GAS or mycoplasma titers at this time.

This vignette highlights the frequent observation of microepisodes, which are best described as brief, milder episodes that often spontaneously remit. As GAS was found by a rapid antigen test in the absence of a sore throat, this could be coincidental. However, GAS pharyngitis presentations often differ in preschool-aged children [33], and the patient's rapid response to antibiotics suggests an immune-modulated pathophysiology. In addition, the effects of GAS exposure by family members are underscored in this case. The absence of titer elevations in this child may have been due to her young age [78].

\section{PANDAS Treatment}

Surgical interventions, including tonsillectomy and/or adenoidectomy, have been considered in children with PANDAS [79-81]. However, a recent study compared GAS titers, PANDAS classification, and OCD and tic severity in subjects with OCD and/or tics who had undergone a tonsillectomy and/or adenoidectomy, and those who had not [82]. No significant differences between GAS titers, PANDAS classification, or symptom severity of surgical and nonsurgical groups were found. These results provide support that tonsillectomies and/or adenoidectomies do not prevent PANDAS symptoms and may even increase susceptibility by altering immune defenses. However, those with clear medical indications (e.g., obstructive sleep apnea; chronic, treatmentresistant GAS pharyngitis) should be treated according to current guidelines for tonsillectomies [83]. A case report noted that a patient with PANDAS who exhibited chronic GAS infections underwent a tonsillectomy and at one-year follow- 
up had not experienced infection or PANDAS symptoms since surgery [79].

Antibiotics have been shown as often resulting in symptom alleviation in some youth with PANDAS [7••, 10, 84]. Cefdinir has been recently investigated as a potential treatment for PANDAS, and it was found that youth with PANDAS who were treated with cefdinir exhibited improvements in tic symptom severity compared to the group receiving placebo. Some improvement was also displayed in OCD symptom severity [85]. A study is currently underway investigating azithromycin as a PANDAS treatment. Earlier studies have supported antibiotics during active infection [10, 12], and mixed results exist regarding efficacy as prophylaxis [84, 86]. Plasmapheresis has been investigated as a potential PANDAS treatment, with results demonstrating improvement in OCD symptoms at one-month and 12-month follow-up [87]. However, due to the limited number of studies, the plasmapheresis guidelines state that there is "insufficient evidence to support or refute the use of plasmapheresis" in PANDAS [88]. Due to the medical risks involved with this treatment, this option is best reserved for those with severe and impairing symptoms not unlike those seen in autoimmune encephalitis. Another treatment option includes intravenous immunoglobulin (IVIG), which was found to be superior to placebo in a randomized clinical trial for youth with PANDAS [87], and effective in case reports [39, 89]. In contrast, IVIG was not effective for adult patients with tics [90]. Steroids have been effective in tic reduction in a case of two youths with TS [91], but clinical trials are necessary to further investigate this treatment. A randomized double-blind clinical trial found fluoxetine therapy augmented with celecoxib, a nonsteroid anti-inflammatory, to be effective in OCD symptom reduction in adults compared to fluoxetine and placebo [92].

\section{Future Research}

There still exists inconsistency in the application of the PANDAS diagnostic criteria, as noted by the use of singlepoint-in-time detection of antibody titers at the upper limit of normal range, resulting in false-positive PANDAS cases being unnecessarily treated with antibiotics [93]. However, while the issue of diagnostic integrity persists, it is difficult to diagnose appropriately when different iterations of infectiontriggered OCD and tics are in existence and reliable biological markers are lacking. Notably, the diagnosis of PANDAS, similar to that of Sydenham's chorea, is based on clinical data rather than diagnostic markers. Overall, there is a need for diagnostic measures, reliable diagnostic biomarkers, and clear consensus on diagnostic iterations. Theories regarding the pathogenesis of PANDAS are still in debate; microbes have been proposed to induce autoantibodies that bind to brain proteins and affect behavior [65]. However, empirical studies are needed to further investigate this theory. As illustrated by recent research, immune-modulatory treatment is an area that requires further investigation, as well as the development of clear guidelines on when to implement antibiotic therapy and which class of antibiotic to use. Overall, the classic PANDAS phenotype has shown some consistencies across the existing literature and this presentation is perhaps less controversial. However, the clinical criteria are likely to undergo additional iterations as discoveries are made regarding biological markers, effective treatments, and pathophysiology.

\section{Compliance with Ethics Guidelines}

Conflict of Interest Tanya K. Murphy received research support from Shire, Pfizer Pharmaceuticals, Otsuka Pharmaceuticals, Transcept Pharmaceuticals, Inc., AstraZeneca Neuroscience iMED, Sunovion Pharmaceuticals, NIH, Ortho McNeil Scientific Affairs, International OCD Foundation, Tourette Syndrome Association, All Children's Hospital Research Foundation, and Centers for Disease Control; serves for the advisory boards of Tourette Syndrome Association, Scientific Advisory Board of IOCDF, and for the PANDAS Network; received textbook honorarium from Lawrence Erlbaum.

Diana M. Gerardi, and E. Carla Parker-Athill declare that they have no conflict of interest.

Human and Animal Rights and Informed Consent This article does not contain any studies with human or animal subjects performed by any of the authors

\section{References}

Papers of particular interest, published recently, have been highlighted as:

- Of importance

-• Of major importance

1. Swedo SE, Leonard HL, Garvey M, et al. Pediatric autoimmune neuropsychiatric disorders associated with streptococcal infections: clinical description of the first 50 cases. Am J Psychiatry. 1998; 155: 264-71.

2. Swedo SE, Leonard HL, Rapoport JL. The pediatric autoimmune neuropsychiatric disorders associated with streptococcal infection (PANDAS) subgroup: separating fact from fiction. Pediatrics. 2004;113:907-11.

3. Kurlan R, Kaplan EL. The pediatric autoimmune neuropsychiatric disorders associated with streptococcal infection (PANDAS) etiology for tics and obsessive-compulsive symptoms: hypothesis or entity? Practical considerations for the clinician. Pediatrics. 2004;113:883-6.

4.• Swedo SE, Leckman J, Rose N. From research subgroup to clinical syndrome: modifying the PANDAS criteria to describe PANS. Pediatr Ther. 2012;2(113):2. This publication identifies a new iteration of PANDAS and contains a descriptive summary of the classic phenotypic presentation.

5.• Singer HS, Gilbert DL, Wolf DS, Mink JW, Kurlan R. Moving from PANDAS to CANS. J Pediatr. 2012;160:725-31. This paper yields a new iteration of PANDAS with suggestions of differential diagnosis and possible testing. 
6. Bernstein GA, Victor AM, Pipal AJ, Williams KA. Comparison of clinical characteristics of pediatric autoimmune neuropsychiatric disorders associated with streptococcal infections and childhood obsessive-compulsive disorder. J Child Adolesc Psychopharmacol. 2010;20:333-40.

7.• Murphy TK, Storch EA, Lewin AB, Edge PJ, Goodman WK. Clinical factors associated with pediatric autoimmune neuropsychiatric disorders associated with streptococcal infections. J Pediatr. 2012;160:314-9. This publication adds to the literature by describing characteristics of youth with PANDAS OCD compared to nonPANDAS OCD.

8. Mell LK, Davis RL, Owens D. Association between streptococcal infection and obsessive-compulsive disorder, Tourette's syndrome, and tic disorder. Pediatrics. 2005;116:56-60.

9. Murphy TK, Sajid M, Soto O, et al. Detecting pediatric autoimmune neuropsychiatric disorders associated with streptococcus in children with obsessive-compulsive disorder and tics. Biol Psychiatry. 2004;55:61-8.

10. Murphy ML, Pichichero ME. Prospective identification and treatment of children with pediatric autoimmune neuropsychiatric disorder associated with group A streptococcal infection (PANDAS). Arch Pediatr Adolesc Med. 2002;156:356.

11. Murphy TK, Storch E, Turner A, Reid J, Tan J, Lewin A. Maternal history of autoimmune disease in children presenting with tics and/or obsessive-compulsive disorder. J Neuroimmunol. 2010;229:243-7.

12. Falcini F, Lepri G, Rigante D, Bertini F, Cerinic MM. PReSFINAL-2252: descriptive analysis of pediatric autoimmune neuropsychiatric disorder associated with streptococcus infection (PANDAS) in a cohort of 65 Italian patients. Pediatr Rheumatol J. 2013;11 Suppl 2:242.

13. Lougee L, Perlmutter SJ, Nicolson R, Garvey MA, Swedo SE. Psychiatric disorders in first-degree relatives of children with pediatric autoimmune neuropsychiatric disorders associated with streptococcal infections (PANDAS). J Am Acad Child Adolesc Psychiatry. 2000;39:1120-6.

14. Lewin AB, Storch EA, Murphy TK. Pediatric autoimmune neuropsychiatric disorders associated with streptococcus in identical siblings. J Child Adolesc Psychopharmacol. 2011;21:177-82.

15. Dranitzki Z, Steiner I. PANDAS in siblings: a common risk? Eur J Neurol. 2007;14(6):e4.

16. Lewin AB, Storch EA, Mutch PJ, Murphy TK. Neurocognitive functioning in youth with pediatric autoimmune neuropsychiatric disorders associated with streptococcus. J Neuropsychiatry Clin Neurosci. 2011;23:391-8.

17. Hirschtritt ME, Hammond CJ, Luckenbaugh D, et al. Executive and attention functioning among children in the PANDAS subgroup. Child Neuropsychol. 2009;15:179-94.

18. Langlois M, Force L. Nosologic and clinical revision of Gilles de la Tourette disease evoked by the action of certain neuroloeptics on its course. Rev Neurol (Paris). 1965;113:641-5.

19. Cardona F, Orefici G. Group A streptococcal infections and tic disorders in an Italian pediatric population. J Pediatr. 2001;138: $71-5$.

20. Müller N, Riedel M, Blendinger C, Forderreuther S, Abele-Horn M. Childhood Tourette's syndrome and infection with mycoplasma pneumoniae. Am J Psychiatry. 2000;157:481-2.

21. Murphy TK, Goodman WK, Fudge MW, et al. B lymphocyte antigen D8/17: a peripheral marker for childhood-onset obsessivecompulsive disorder and Tourette's syndrome? Am J Psychiatry. 1997;154:402-7.

22. Loiselle CR, Wendlandt JT, Rohde CA, Singer HS. Antistreptococcal, neuronal, and nuclear antibodies in Tourette syndrome. Pediatr Neurol. 2003;28:119-25.

23. Singer HS, Giuliano JD, Hansen BH, et al. Antibodies against a neuron-like (HTB-10 neuroblastoma) cell in children with Tourette syndrome. Biol Psychiatry. 1999;46:775-80.
24. Martino D, Chiarotti F, Buttiglione M, et al. The relationship between group A streptococcal infections and Tourette syndrome: a study on a large service-based cohort. Dev Med Child Neurol. 2011;53:951-7. This paper contributes to the growing body of literature that supports an association between GAS infections and TS.

25. Morris CM, Pardo-Villamizar C, Gause CD, Singer HS. Serum autoantibodies measured by immunofluorescence confirm a failure to differentiate PANDAS and Tourette syndrome from controls. J Neurol Sci. 2009;276:45-8.

26. Kurlan R, Johnson D, Kaplan EL. Streptococcal infection and exacerbations of childhood tics and obsessive-compulsive symptoms: a prospective blinded cohort study. Pediatrics. 2008;121: 1188-97.

27. Leckman JF, King RA, Gilbert DL, et al. Streptococcal upper respiratory tract infections and exacerbations of tic and obsessivecompulsive symptoms: a prospective longitudinal study. J Am Acad Child Adolesc Psychiatry. 2011;50:108-18.e3.

28. Luo F, Leckman JF, Katsovich L, et al. Prospective longitudinal study of children with tic disorders and/or obsessive-compulsive disorder: relationship of symptom exacerbations to newly acquired streptococcal infections. Pediatrics. 2004;113:e578-85.

29. Schrag A, Gilbert R, Giovannoni G, Robertson M, Metcalfe C, Ben-Shlomo Y. Streptococcal infection, Tourette syndrome, and OCD: is there a connection? Neurology. 2009;73:1256-63.

30. Kiessling LS, Marcotte AC, Culpepper L. Antineuronal antibodies in movement disorders. Pediatrics. 1993;92:39-43.

31. Berrios X, Quesney F, Morales A, Blazquez J, Bisno AL. Are all recurrences of "pure" Sydenham chorea true recurrences of acute rheumatic fever? J Pediatr. 1985;107:867-72.

32. Swedo SE, Leonard HL. Childhood movement disorders and obsessive compulsive disorder. J Clin Psychiatry. 1994;55(suppl):32-7.

33. Schwartz RH, Hayden GF, Wientzen R. Children less than threeyears-old with pharyngitis are group A streptococci really that uncommon? Clin Pediatr (Phila). 1986;25(4):185-8.

34. Schwartz RH, Wientzen RL, Pedreira F, Feroli EJ, Mella GW, Guandolo VL. Penicillin V for group A streptococcal pharyngotonsillitis: a randomized trial of seven vs ten days' therapy. JAMA. 1981;246:1790-5.

35. Toufexis M, DeOleo C, Elia J, Murphy TK. A link between perianal strep and pediatric autoimmune neuropsychiatric disorder associated with streptococcal infection (PANDAS). J Neuropsychiatry Clin Neurosci. 2014;26:164-8.

36. Mazon A, Gil-Setas A, Sota de la Gandara LJ, Vindel A, Saez-Nieto JA. Transmission of streptococcus pyogenes causing successive infections in a family. Clin Microbiol Infect. 2003;9:554-9.

37. Petersen JP, Kaltoft MS, Misfeldt JC, Schumacher H, Schonheyder HC. Community outbreak of perianal group A streptococcal infection in Denmark. Pediatr Infect Dis J. 2003;22:105-9.

38. Hoekstra PJ, Manson WL, Steenhuis M-P, Kallenberg CGM, Minderaa RB. Association of common cold with exacerbations in pediatric but not adult patients with tic disorder: a prospective longitudinal study. J Child Adolesc Psychopharmacol. 2005;15: 285-92.

39. Allen AJ, Leonard HL, Swedo SE. Case study: a new infectiontriggered, autoimmune subtype of pediatric OCD and Tourette's syndrome. J Am Acad Child Adolesc Psychiatry. 1995;34:307-11.

40. Edwards MJ, Dale RC, Church AJ, et al. Adult-onset tic disorder, motor stereotypies, and behavioural disturbance associated with antibasal ganglia antibodies. Mov Disord. 2004;19:1190-6.

41. Yiș U, Kurul SH, Çakmakçı H, Dirik E. Mycoplasma pneumoniae: nervous system complications in childhood and review of the literature. Eur J Pediatr. 2008;167:973-8.

42. Müller N, Abele-Horn M, Riedel M. Infection with Mycoplasma pneumoniae and Tourette's syndrome. Psychiatry Res. 2004;129: 119-25. 
43. Rhee H, Cameron DJ. Lyme disease and pediatric autoimmune neuropsychiatric disorders associated with streptococcal infections (PANDAS): an overview. Int J Gen Med. 2012;5:163-74.

44. Fallon BA, Nields JA. Lyme disease: a neuropsychiatric illness. Am J Psychiatry. 1994;151:1571-83.

45. Riedel M, Straube A, Schwarz MJ, Wilske B, Muller N. Lyme disease presenting as Tourette's syndrome. Lancet. 1998;351(9100):418-9.

46. Church A, Dale R, Giovannoni G. Anti-basal ganglia antibodies: a possible diagnostic utility in idiopathic movement disorders? Arch Dis Child. 2004;89:611-4.

47. Pavone P, Bianchini R, Parano E, et al. Anti-brain antibodies in PANDAS versus uncomplicated streptococcal infection. Pediatr Neurol. 2004;30:107-10.

48. Kirvan CA, Swedo SE, Snider LA, Cunningham MW. Antibodymediated neuronal cell signaling in behavior and movement disorders. J Neuroimmunol. 2006;179:173-9.

49. Ben-Pazi H, Stoner JA, Cunningham MW. Dopamine receptor autoantibodies correlate with symptoms in Sydenham's chorea. PLoS One. 2013;8(9):e73516.

50. Church A, Dale RC. Antistreptolysin-O titers: implications for adult PANDAS. Am J Psychiatry. 2002;159:320

51. Morris-Berry C, Pollard M, Gao S, Thompson C, Singer H. Antistreptococcal, tubulin, and dopamine receptor 2 antibodies in children with PANDAS and Tourette syndrome: single-point and longitudinal assessments. J Neuroimmunol. 2013;264:106-13.

52. Singer HS, Hong JJ, Yoon DY, Williams PN. Serum autoantibodies do not differentiate PANDAS and Tourette syndrome from controls. Neurology. 2005;65:1701-7.

53. Brilot F, Merheb V, Ding A, Murphy T, Dale R. Antibody binding to neuronal surface in Sydenham chorea, but not in PANDAS or Tourette syndrome. Neurology. 2011;76:1508-13.

54. Gabbay V, Coffey BJ, Guttman LE, et al. A cytokine study in children and adolescents with Tourette's disorder. Prog Neuropsychopharmacol Biol Psychiatry. 2009;33:967-71.

55. Bombaci M, Grifantini R, Mora M, et al. Protein array profiling of tic patient sera reveals a broad range and enhanced immune response against Group A Streptococcus antigens. PLoS One. 2009;4(7):e6332.

56. Hounie AG, Cappi C, Cordeiro Q, et al. TNF-alpha polymorphisms are associated with obsessive-compulsive disorder. Neurosci Lett. 2008;442:86-90.

57. Cappi C, Muniz RK, Sampaio AS, et al. Association study between functional polymorphisms in the TNF-alpha gene and obsessivecompulsive disorder. Arq Neuropsiquiatr. 2012;70:87-90.

58. Luleyap HU, Onatoglu D, Yilmaz MB, et al. Association between pediatric autoimmune neuropsychiatric disorders associated with streptococcal infections disease and tumor necrosis factor- $\alpha$ gene$308 \mathrm{~g} / \mathrm{a},-850 \mathrm{c} / \mathrm{t}$ polymorphisms in 4-12-year-old children in Adana/Turkey. Indian J Hum Genet. 2013;19:196-201.

59. Zhang D, Patel A, Zhu Y, Siegel A, Zalcman SS. Anti-streptococcus IgM antibodies induce repetitive stereotyped movements: cell activation and co-localization with $\mathrm{Fc} \alpha / \mu$ receptors in the striatum and motor cortex. Brain Behav Immun. 2012;26:521-33.

60. Parameswaran N, Patial S. Tumor necrosis factor- $\alpha$ signaling in macrophages. Crit Rev Eukaryot Gene Expr. 2010;20:87-103.

61. Chomarat P, Dantin C, Bennett L, Banchereau J, Palucka AK. TNF skews monocyte differentiation from macrophages to dendritic cells. J Immunol. 2003;171:2262-9.

62. Miro K, Alghanmi R, Rankin J, Geng L, Jyonouchi H. Immune abnormalities in pediatric acute-onset neuropsychiatric syndrome (PANS). J Allergy Clin Immunol. 2013;131(2):AB67.

63. Murphy TK, Husted DS, Edge PJ. Preclinical/Clinical evidence of central nervous system infectious etiology in PANDAS. Adv Neurol. 2005;99:148-58.
64. Brimberg L, Benhar I, Mascaro-Blanco A, et al. Behavioral, pharmacological, and immunological abnormalities after streptococcal exposure: a novel rat model of Sydenham chorea and related neuropsychiatric disorders. Neuropsychopharmacology. 2012;37: 2076-87. This supports the hypothesis of GAS involvement in the development of neuropsychiatric disorder symptoms.

65. Hornig M. The role of microbes and autoimmunity in the pathogenesis of neuropsychiatric illness. Curr Opin Rheumatol. 2013;25: 488-95.

66. Yaddanapudi K, Hornig M, Serge R, et al. Passive transfer of streptococcus-induced antibodies reproduces behavioral disturbances in a mouse model of pediatric autoimmune neuropsychiatric disorders associated with streptococcal infection. Mol Psychiatry. 2009;15:712-26.

67. Cox CJ, Sharma M, Leckman JF, et al. Brain human monoclonal autoantibody from Sydenham chorea targets dopaminergic neurons in transgenic mice and signals dopamine D2 receptor: implications in human disease. J Immunol. 2013;191:5524 41.

68. Nordstrom E, Burton F. A transgenic model of comorbid Tourette's syndrome and obsessive-compulsive disorder circuitry. Mol Psychiatry. 2001;7:617-25.

69. Kirvan CA, Swedo SE, Heuser JS, Cunningham MW. Mimicry and autoantibody-mediated neuronal cell signaling in Sydenham chorea. Nat Med. 2003;9:914-20.

70. Das A, Radhakrishnan A. A case of PANDAS with Kleine-Levin type periodic hypersomnia. Sleep Med. 2012;13:319-20.

71. Ray PC, Tas DA, Gul Celik G, Yolga Tahiroglu A, Avci A, Erken E. Periodic fever and hyperimmunoglobulin D syndrome in a boy with pediatric autoimmune neuropsychiatric disorders associated with group A $\beta$-Hemolytic streptococcus. J Child Adolesc Psychopharmacol. 2013;23:302-4.

72. Bejerot $\mathrm{S}$, Hesselmark E, Wallén $\mathrm{H}$, et al. Pediatric autoimmune neuropsychiatric disorders associated with streptococcal infections perceived as conversion disorder: a case study of a young woman. Brain Behav Immun. 2013;32:e16-7.

73. Calkin CV, Carandang CG. Certain eating disorders may be a neuropsychiatric manifestation of PANDAS: case report. J Can Acad Child Adolesc Psychiatry. 2007;16:132.

74. Sokol MS. Infection-triggered anorexia nervosa in children: clinical description of four cases. J Child Adolesc Psychopharmacol. 2000;10:133-45.

75. Sokol MS, Gray NS. Case study: an infection-triggered, autoimmune subtype of anorexia nervosa. J Am Acad Child Adolesc Psychiatry. 1997;36:1128-33

76. Rubenstein CS, Pigott TA, L'Heureux F, Hill JL. A preliminary investigation of the lifetime prevalence of anorexia and bulimia nervosa in patients with obsessive compulsive disorder. J Clin Psychiatry. 1992;53:309-14.

77. Maguire GA, Viele SN, Agarwal S, Handler E, Franklin D. Stuttering onset associated with streptococcal infection: a case suggesting stuttering as PANDAS. Ann Clin Psychiatry. 2010;22: 283-4.

78. Renneberg J, Soderstrom M, Prellner K, Forsgren A, Christensen P. Age-related variations in anti-streptococcal antibody levels. Eur J Clin Microbiol Infect Dis. 1989;8:792-5.

79. Alexander AA, Patel NJ, Southammakosane CA, Mortensen MM Pediatric autoimmune neuropsychiatric disorders associated with streptococcal infections (PANDAS): an indication for tonsillectomy. Int J Pediatr Otorhinolaryngol. 2011;75:872-3.

80. Batuecas Caletrío Á, Sánchez González F, Cruz Ruiz SS, Santos Gorjón P, Blanco Pérez P. PANDAS syndrome: a new tonsillectomy indication? Acta Otorrinolaringol. 2008;59:362-3.

81. Heubi C, Shott SR. PANDAS: pediatric autoimmune neuropsychiatric disorders associated with streptococcal infections - an uncommon, but important indication for tonsillectomy. Int J Pediatr Otorhinolaryngol. 2003;67:837-40. 
82. Murphy TK, Lewin AB, Parker-Athill EC, Storch EA, Mutch PJ. Tonsillectomies and adenoidectomies do not prevent the onset of pediatric autoimmune neuropsychiatric disorder associated with group A streptococcus. Pediatr Infect Dis J. 2013;32:834-8.

83. Baugh RF, Archer SM, Mitchell RB, et al. Clinical practice guideline tonsillectomy in children. Otolaryngol Head Neck Surg. 2011;144:S1-30.

84. Snider LA, Lougee L, Slattery M, Grant P, Swedo SE. Antibiotic prophylaxis with azithromycin or penicillin for childhood-onset neuropsychiatric disorders. Biol Psychiatry. 2005;57:788-92.

85. Murphy TK, Parker-Athill EC, Lewin AB, Storch EA, Mutch PJ. Cefdinir for new onset pediatric neuropsychiatric disorders: a pilot randomized trial. J Child Adolesc Psychopharmacol. in press.

86. Garvey MA, Perlmutter SJ, Allen AJ, et al. A pilot study of penicillin prophylaxis for neuropsychiatric exacerbations triggered by streptococcal infections. Biol Psychiatry. 1999;45:1564-71.

87. Perlmutter SJ, Leitman SF, Garvey MA, et al. Therapeutic plasma exchange and intravenous immunoglobulin for obsessivecompulsive disorder and tic disorders in childhood. Lancet. 1999;354(9185):1153-8.

88. Cortese I, Chaudhry V, So Y, Cantor F, Cornblath D, Rae-Grant A. Evidence-based guideline update: plasmapheresis in neurologic disorders: report of the Therapeutics and Technology Assessment Subcommittee of the American Academy of Neurology. Neurology. 2011;76:294-300.

89. Elia J, Dell ML, Friedman DF, et al. PANDAS with catatonia: a case report. Therapeutic response to lorazepam and plasmapheresis. J Am Acad Child Adolesc Psychiatry. 2005;44:1145-50.

90. Hoekstra PJ, Minderaa RB, Kallenberg C. Lack of effect of intravenous immunoglobulins on tics: a double-blind placebo-controlled study. J Clin Psychiatry. 2004;65: $537-42$.

91. Matarazzo EB. Tourette's syndrome treated with ACTH and prednisone: report of two cases. J Child Adolesc Psychopharmacol. 1992;2:215-26.

92. Sayyah M, Boostani H, Pakseresht S, Malayeri A. A preliminary randomized double-blind clinical trial on the efficacy of celecoxib as an adjunct in the treatment of obsessive-compulsive disorder. Psychiatry Res. 2011;189:403-6.

93. Gabbay V, Coffey BJ, Babb JS, et al. Pediatric autoimmune neuropsychiatric disorders associated with streptococcus: comparison of diagnosis and treatment in the community and at a specialty clinic. Pediatrics. 2008;122:273-8. 